\title{
OPTIMIZED TRIAD ALGORITHM \\ FOR \\ ATTITUDE DETERMINATION
}

\section{$13: 510$}

\author{
Itzhack Y. Bar-Itzhack $\quad$ Richard R. Harman \\ Flight Dynamics Support Branch, Code 553 \\ NASA Goddard Space Flight Center \\ Greenbelt, MD 20771
}

\begin{abstract}
TRIAD is a well known simple algorithm that generates the attitude matrix between two coordinate systems when the components of two abstract vectors are given in the two systems. TRIAD, however, is sensitive to the order at which the algorithm handles the vectors, such that the resulting attitude matrix is influenced more by the vector proccessed first.

In this work we present a new algorithm, which we call Optimized TRIAD, that blends, in a specified manner, the two matrices generated by TRIAD when processing one vector first, and then when processing the other vector first. On the average, Optimized TRIAD yields a matrix which is better than either one of the two matrices in that it is the closest to the correct matrix. This result is demonstrated through simulation.
\end{abstract}

\section{BACKGROUND}

When the components of two abstract vectors are given in two different coordinate systems, it is possible to find the orientation difference between the two systems. In particular, we can casily find the transformation matrix from one coordinate system to the other. TRIAD ${ }^{1,2}$ is an algorithm that does just that. The process of finding the matrix using TRIAD is as follows. Let $w_{1}$ and $w_{2}$ denote the column matrices whose elements are, respectively, the components of the two abstract vectors when resolved in body coordinates, and let $v_{1}$ and $v_{2}$ denote, respectively, the two column matrices whose elements are the components of the abstract vectors when resolved in the other, usually reference, system. The algorithm calls for the computation of the following column matrices in body coordinates:

$$
\begin{gathered}
r_{1}=w_{1} /\left|w_{1}\right| \\
r_{2}=\left(r_{1} \times w_{2}\right) /\left|r_{1} \times w_{2}\right| \\
r_{3}=r_{1} \times r_{2}
\end{gathered}
$$

\footnotetext{
* Sophie and William Shamban Profeseor of Aerospace Engineering. On abbatical leave from the Faculty of Aerospace Engineering. Technion-Imel Instinute of Technology, Haifn 32000, Irrael. Member Technion Space Research Instinte. IEEE Fellow.
}

Acrospace Engineer.

This work was performed on \& Natioanl Research Council NASA Research Aseocinteship. 
and the following corresponding column matrices in the reference system:

$$
\begin{gathered}
s_{1}=v_{1} /\left|v_{1}\right| \\
s_{2}=\left(s_{1} \times v_{2}\right) /\left|s_{1} \times v_{2}\right| \\
s_{3}=s_{1} \times s_{2}
\end{gathered}
$$

Then the attitude matrix that transforms from body to the reference coordinate system is computed as follows:

$$
A=r_{1} \cdot s_{1}^{T}+r_{2} \cdot s_{2}^{T}+r_{3} \cdot s_{3}^{T}
$$

where $\mathrm{T}$ denotes the transpose.

Following the process indicated in (1) and (2), we realize that the vector which is designated as first, is normalized, but other than that, remains intact, whereas the other vector serves as a means to define the second vector in the triad pair which determines the attitude. There is, therefore, an uneven consideration of the two vectors where the first is given a preference in the determination of A. We say that the first vector serves as an anchor in the computation of the transformation matrix. It is, indeed, a good engineering practice to use the vector measured by the most accurate device as the anchor vector. For example, it is very logical to use the vector measured by a star tracker as anchor when the other vector is measured by magnetometers. One may wonder though whether this is the best one can do. We maintain that we can do better, and propose a TRIAD-based algorithm which yields better results. This algorithm, which we name Optimized TRIAD, is introduced next.

\section{THE OPTIMIZED TRIAD}

The accuracy of each vector-measuring device is quantified by the standard deviation of its error. Accordingly, the vector measured by a star tracker is assigned a standard deviation smaller than that assigned to a magnetometer, for example. Borrowing this notion, we assign a standard deviation to the TRIAD-computed attitude matrix that corresponds to the standard deviation of the anchor vector used in computing the matrix. Therefore, the attitude matrix $A_{1}$, in whose computation vector no. 1 is used as anchor, is assigned the standard deviation $\sigma_{1}$, which is the the standard deviation of vector no. 1 . Similarly, if vector no. 2 serves as anchor, we denote the computed attitude matrix by $A_{2}$ and assign to it the standard deviation $\sigma_{2}$ which is the standard deviation of vector no. 2. Actually, since the computation which yields the matrix is nonlinear and is based on both vectors, there is no simple linear relation between the standard deviation of the anchor vector and that of the resulting matrix, but since we are concerned only with the relative accuracy of $A_{1}$ and $A_{2}$, the expression of their accuracy by $\sigma_{1}$ and $\sigma_{2}$ respectively, fits well our final purpose.

It is well known (see the appendix) that when $y_{1}$ and $y_{2}$ are independent unbiased scalar measurement of an unknown scalar, $x$, and their measurement errors have standard deviations $\sigma_{1}$ and $\sigma_{2}$, respectively, then $\hat{x}$, the linear unbiased minimum variance estimate of $x$, is given by:

$$
\hat{x}=\frac{\sigma_{2}^{2}}{\sigma_{1}^{2}+\sigma_{2}^{2}} y_{1}+\frac{\sigma_{1}^{2}}{\sigma_{1}^{2}+\sigma_{2}^{2}} y_{2}
$$


Following (4), we postulate that given $A_{1}$ with its assigned standard deviation $\sigma_{1}$ and $A_{2}$ with its assigned standard deviation $\sigma_{2}$, we can find $\hat{A}^{\prime}$, an estimate of $A$ which is better than either $A_{1}$ or $A_{2}$, when using the estimator of (4); that is,

$$
\hat{A}^{\prime}=\frac{\sigma_{2}^{2}}{\sigma_{1}^{2}+\sigma_{2}^{2}} A_{1}+\frac{\sigma_{1}^{2}}{\sigma_{1}^{2}+\sigma_{2}^{2}} A_{2}
$$

An interesting aspect of this estimator (as well as that of (4)) is the conclusion that adding some of the worse result to the better, may yield an estimate whose accuracy is greater than that of the better. Since $\hat{\mathrm{A}}^{\prime}$ is a result of the addition of fructions of two orthogonal matrices, $\hat{\mathrm{A}}^{\prime}$ is not necessarily orthogonal, and thus is not a legitimate attitude matrix, unless it is orthogonalized. Since $\hat{A}^{\prime}$ is close to being orthogonal, one orthogonalization cycle, as follows ${ }^{4}$, suffices:

$$
\hat{\mathrm{A}}=0.5\left[\hat{\mathrm{A}}^{\cdot}+\left(\hat{\mathrm{A}}^{-1}\right)^{\mathrm{T}}\right]
$$

It should be noted that the inversion of $\hat{A}^{\prime}$ is an easy task since the inverse of a $3 \times 3$ matrix can be computed analytically. It is cumbersome, if not impossible, to prove analytically that $\hat{\mathrm{A}}$ is better than either $A_{1}$ or $A_{2}$; however, we can try to show it empirically. This is done in the next section.

\section{ALGORITHM TESTING}

\section{III.1 Static Testing}

In the static testing we chose some fixed attitude matrix, $A_{\text {true' }}$ and the components of the unit vectors $v_{1}$ and $v_{2}$ (two abstract vectors resolved in the reference system). Then $A_{\text {true }}$ was used to transform $v_{1}$ and $v_{2}$ to the body system. To each component of the latter we added white measurement noise drawn from a random number generator. The added noise was unbiased and had a standard deviation $\sigma_{1}=0.1$ for the noise added to the components of the transform of $v_{1}$ and $\sigma_{2}=0.2$ for the noise added to the components of the transform of $v_{2}$. The noisy column matrices were designated as $w_{1}$ and $w_{2}$. TRIAD was then applied to the four column matrices as described in the preceding section, once when vector no. 1 was used as anchor and once when the other was used as anchor. This generated the attitude matrices $A_{1}$ and $A_{2}$ respectively, which then were used in (5) to generate $\hat{A}^{\prime}$ that was used in $(6)$ to yield the optimized orthogonal matrix $\hat{A}$. The quaternions corresponding to $A_{\text {true }}, A_{1}, A_{2}$, and $\hat{A}$ were computed and denoted by $q_{t r u e}, q_{1}, q_{2}$, and $\hat{q}$ respectively. The error quaternion of each transformation was computed as follows:

$$
\delta \mathbf{q}=\mathbf{q}^{-1} \otimes \mathbf{q}_{\text {true }}
$$

When $q$ was $q_{1}$ we obtained $\delta q_{1}$, when $q$ was $q_{2}$ we obtained $\delta q_{2}$, and when it was $\hat{q}$, we obtained $\delta \hat{q}$. (Note that with the choice of (7) for computing the erroneous quatemion, we assume that $\delta q$ is the transformation quaternion from the erroneous to the true coordinate system). Finally, we extracted from each $\delta q$ the corresponding rotation angle $\delta \varphi$. We thus have expressed the error in the computation of the attitude by a single angular error. That error was the angle by which the computed coordinate system had to be rotated about the appropriate Euler axis in order to coincide with the true body coordinates.

Since $\delta \varphi$ is a random variable, we ran 100 runs (realizations), each for $60 \mathrm{sec}$ and each starting with a 
different seed. Along the time axis the computation was performed every second. We then averaged the 100 realizations at each time point and obtained the ensemble average of each error, that is, we obtained:

$$
\begin{aligned}
& \delta \bar{\varphi}_{1}\left(t_{k}\right)=\frac{1}{100} \sum_{j=1}^{100} \delta \varphi_{1, j}\left(t_{k}\right) \\
& \delta \bar{\varphi}_{2}\left(t_{k}\right)=\frac{1}{100} \sum_{j=1}^{100} \delta \varphi_{2, j}\left(t_{k}\right) \\
& \delta \hat{\varphi}\left(t_{k}\right)=\frac{1}{100} \sum_{j=1}^{100} \delta \hat{\varphi}_{j}\left(t_{k}\right)
\end{aligned}
$$

where $j$ denotes the number of the realization, and $t_{k}$ denotes the point in time where TRIAD and Optimal TRLAD were performed. The value of $\delta \bar{\varphi}_{1}\left(t_{k}\right), \delta \bar{\varphi}_{2}\left(t_{k}\right)$ and $\delta \bar{\varphi}\left(t_{k}\right)$ as function of $t_{k}$ is presented in Fig. (1). We see that $\delta \bar{\varphi}$ was always the smallest. We also computed the running time average of each

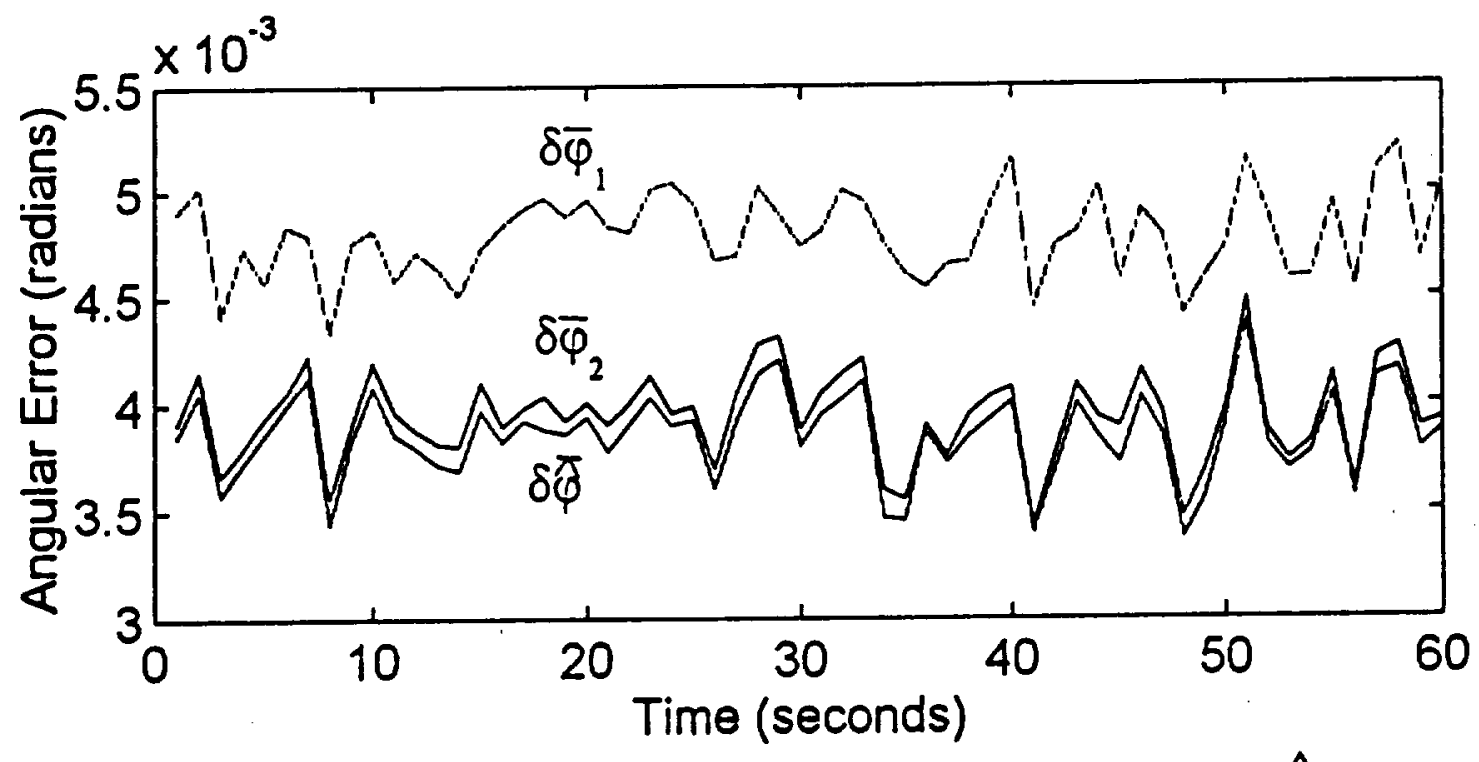

Fig. 1: Ensemble Average of the Error Associated with $A_{1}, A_{2}$, and $\hat{A}$.

ensemble average from the beginning of the run to time $t$. In other words, we computed:

$$
\begin{aligned}
& \delta \bar{\varphi}_{1, a v}\left(t_{k}\right)=\frac{1}{k} \sum_{i=1}^{k} \delta \bar{\varphi}_{1}\left(t_{i}\right) \\
& \delta \bar{\varphi}_{2, a v}\left(t_{k}\right)=\frac{1}{k} \sum_{i=1}^{k} \delta \bar{\varphi}_{2}\left(t_{i}\right)
\end{aligned}
$$




$$
\delta \varphi_{2 v}^{x}\left(t_{k}\right)=\frac{1}{k} \sum_{i=1}^{k} \delta \varphi_{\varphi}^{\pi}\left(t_{i}\right)
$$

The value of $\delta \bar{\varphi}_{1, a v}\left(t_{k}\right), \delta \bar{\varphi}_{2, a v}\left(t_{k}\right)$ and $\delta \bar{\varphi}_{a v}\left(t_{k}\right)$ as function of $t_{k}$ is presented in Fig. 2. It is

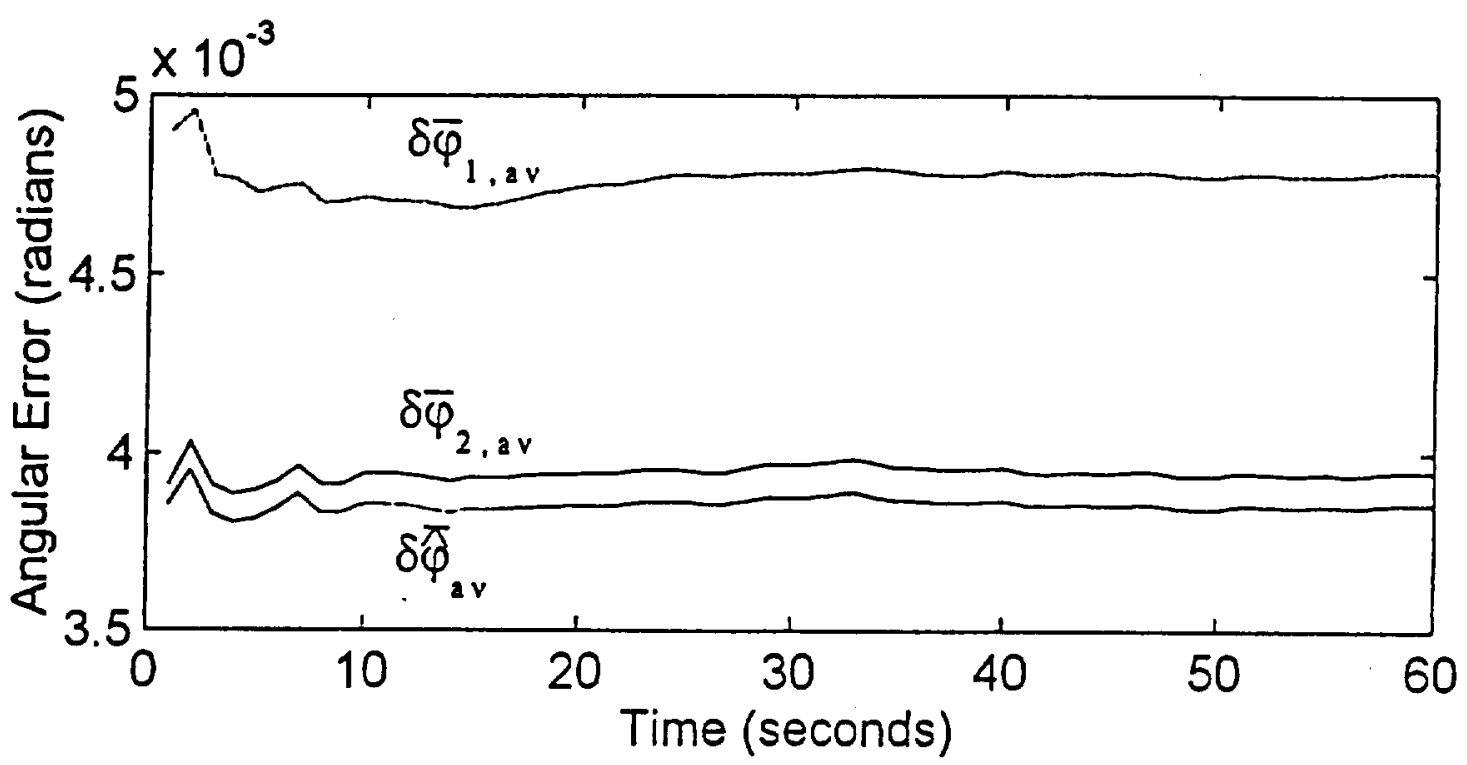

Fig. 2: Running Time Average of the Ensemble Average of the Error Associated with $A_{1}, A_{2}$, and $\hat{A}$.

obvious from Fig. 2 that $\hat{A}$ is superior to either $A_{1}$ or $A_{2}$. In other words, on the average, the Optimized TRIAD yields better results for the case tested.

\section{III.1 Dynamic Testing}

To check the influence of changing attitude, we repeated the same runs and computations as described before, for a changing $A$. The change in $A$ was due to a the body rotation about an axis $\bar{p}$ defined as follows:

$$
\bar{\rho}=\frac{1}{\sqrt{3}}\left[\bar{i}_{b}, \bar{j}_{b}, \bar{k}_{b}\right]
$$

The rotation rate about this axis was $1 \mathrm{pm}$. The graphs of the results of this case, which correspondto those presented in Figs. 1 and 2, are presented in Figs. 3 and 4 respectively. Since the idea behind this algorithm is borrowed from linear estimation theory of independent unbiased measurement errors, one would expect the ensemble average of the angular error to be zero; however, as can be seen in Figs. 1 and 3 , this is not the case. This discrepancy stems from the fact that the displayed error is not linearly related to the averaged matrices. Also, the errors in the computed matrices, $A_{1}$ and $A_{2}$, are not really independent. 


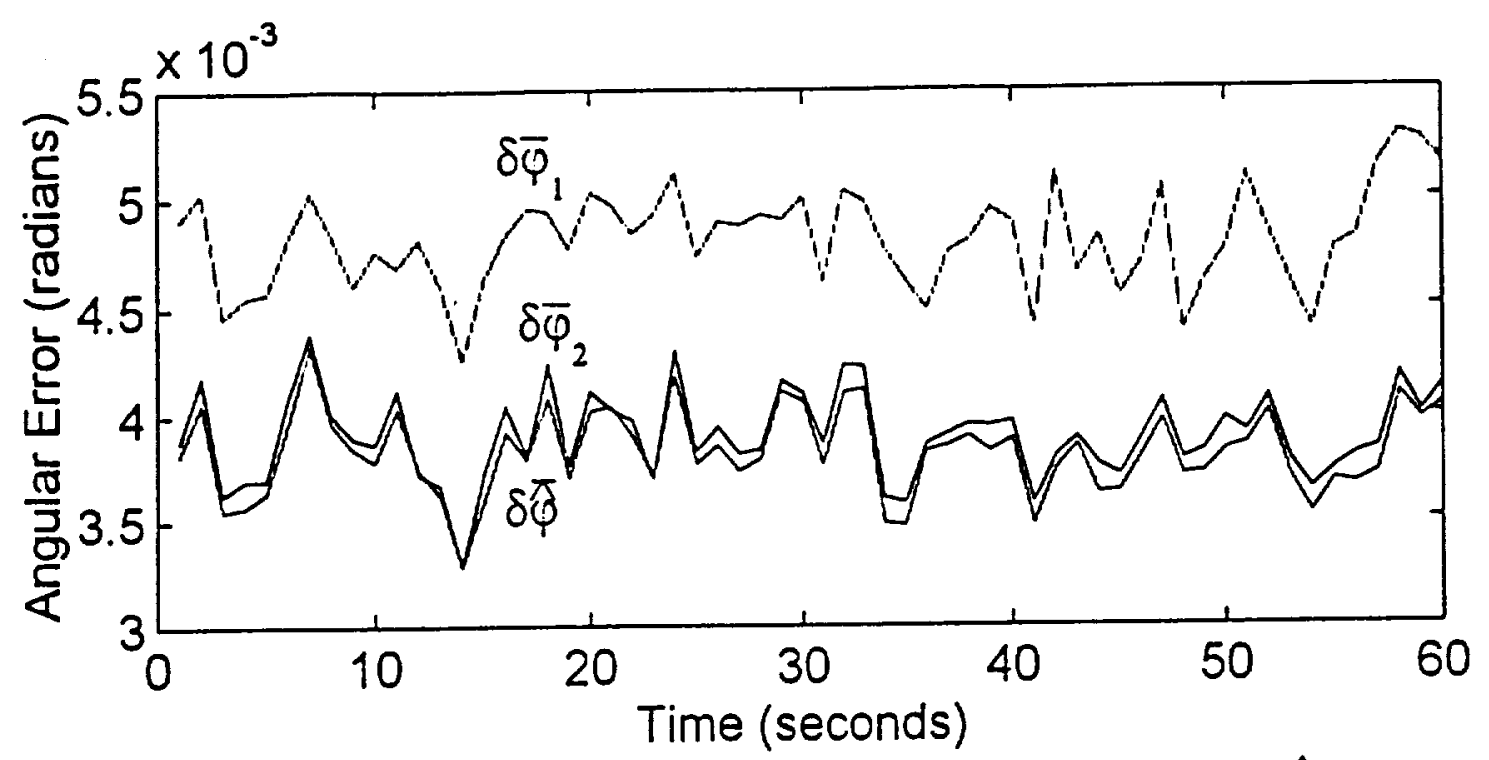

Fig. 3: Ensemble Average of the Error Associated with $A_{1}, A_{2}$, and $\hat{A}$ when $A_{\text {irue }}$ was changing.

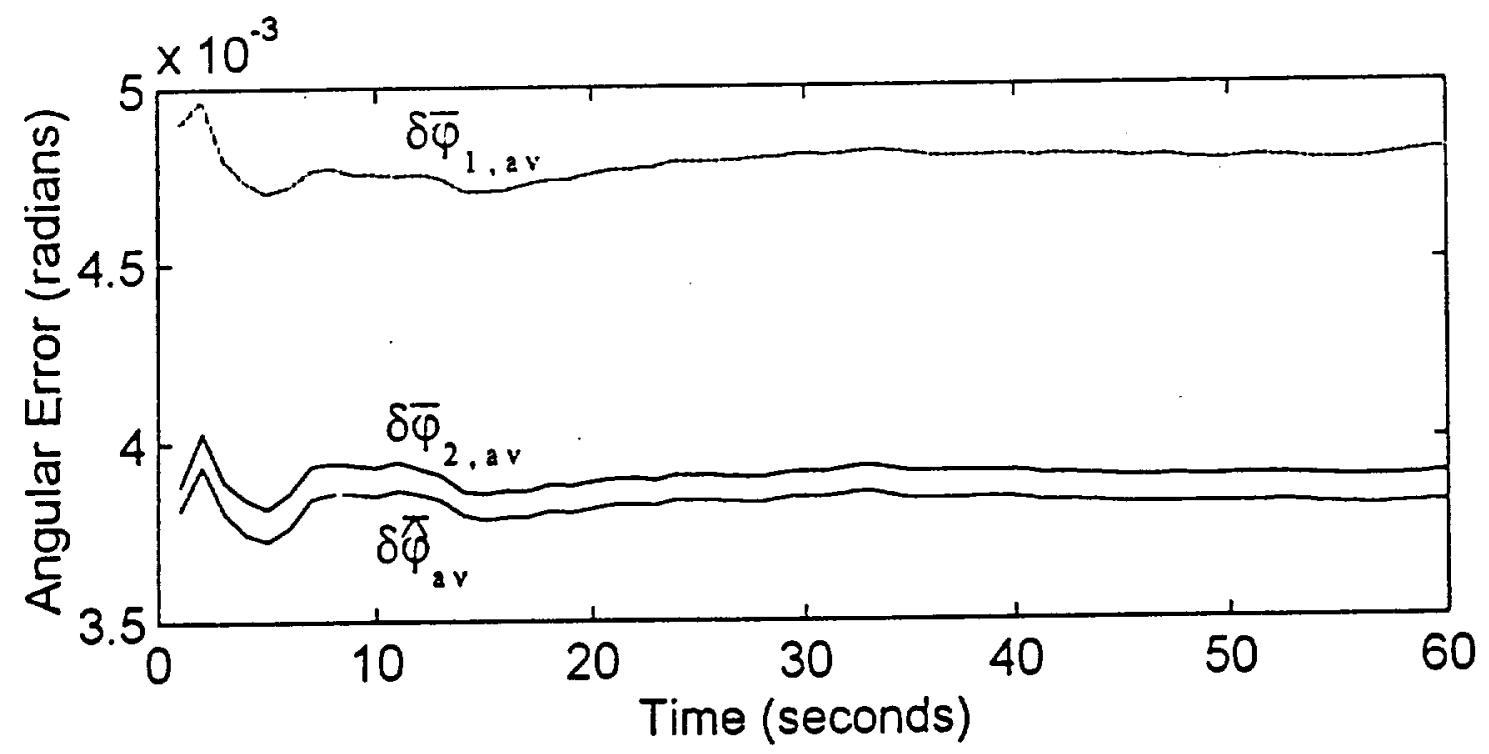

Fig. 4: Running Time Average of the Ensemble Average of the Error Associated with $A_{1}, A_{2}$, and $\hat{A}$ when $A_{\text {true }}$ was changing.

Finally, the vectors $v_{1}$ and $v_{2}$, which are the components of the two abstract vector resolved in the reference coordinates, were constant through all runs. The angle between the two vectors was close to 90 . To investigate the behavior of the algorithm for a different separation angle, we chose two new $\mathbf{v}_{1}$ and $v_{2}$ vectors the angle between which was close $2045^{\circ}$ and ran the last lest case. The results were similar to those presented in Figs. 3 and 4 , only that, as expected, the errors of all three algorithms were nearly $25 \%$ higher. 


\section{CONCLUSIONS}

In this work we have presented a simple TRIAD-based algorithm, which we call Optimized TRIAD, that performs better than TRIAD itself. The algorithm consists of runing TRIAD twice, once with one vector as anchor and once with the other vector as anchor, and weight averaging of the two resultant matrices followed by one orthogonalization cycle. The weights are determined by the accuracy of the measuring devices that produced the vector measurements. The idea behind this algorithm is borrowed from linear estimation theory of independent unbiased measurement errors. However, although the blending of the two TRIAD-generated matrices is based on an unbiased minimum variance formula, the ensemble average of the angular error is not zero, as can be seen in Figs. 1 and 3. This, however, is no surprise, because the displayed error is not linearly related to the averaged matrices. Also, the errors in the computed matrices, $A_{1}$ and $A_{2}$, are not really independent.

We have shown empirically that, indeed, the accuracy of the Optimized TRIAD is better than that of TRIAD even when the latter uses the vector measured most accurately as anchor. It should be noted though that in this statement we refer to the average performance. That is to say that occationally TRIAD may yield results which are better than those obtained using Optimized TRIAD, but on the average, Optimal TRIAD performs better. It is interesting to note that like in Kalman filtering, the correct blending of the better TRIAD-generated attitude matrix with the worse, yields, on the average, a result which is more accurate than the better.

\section{Appendix}

Theorem: Given $y_{1}$ and $y_{2}$ where:

and

$$
\begin{aligned}
& y_{1}=x+v_{1} \\
& y_{2}=x+v_{2}
\end{aligned}
$$

$$
\begin{array}{lll}
E\left\{v_{1}\right]=0 & (2 . a) & E\left\{v_{2}\right\}=0 \\
E\left\{v_{1}^{2}\right\}=\sigma_{1}^{2} & \text { (2.b) } & E\left[v_{2}^{2}\right\}=\sigma_{2}^{2}
\end{array}
$$

Then, the following linear estimator:

$$
\hat{x}=\frac{\sigma_{2}^{2}}{\sigma_{1}^{2}+\sigma_{2}^{2}} y_{1}+\frac{\sigma_{1}^{2}}{\sigma_{1}^{2}+\sigma_{2}^{2}} y_{2}
$$

yields an unbiased minimum variance estimate of $x$.

Proof:

A linear estimator of $\mathrm{x}$ has the form:

$$
\hat{x}=k_{1} y_{1}+k_{2} y_{2}
$$

Substitution of (1) into (4) yields:

$$
\hat{x}=\left(k_{1}+k_{2}\right) x+k_{1} v_{1}+k_{2} v_{2}
$$


Using (5) we can write the estimation error as follos:

$$
e \triangleq x-\hat{x}=\left(1-k_{1}-k_{2}\right) x-k_{1} v_{1}-k_{2} v_{2}
$$

Due to the unbiasedness of $v_{1}$ and $v_{2}$ expressed in $(2 . a, c)$, we obtain from (6):

$$
E[e]=\left(1-k_{1}-k_{2}\right) x
$$

For the estimate to be unbiased, $E[e]$ has to vanish, which yields the necessary condition for unbiasedness:

$$
k_{1}=1-k_{2}
$$

Using (8), (4) becomes:

$$
\hat{x}=\left(1-k_{2}\right) y_{1}+k_{2} y_{2}
$$

and (6) becomes:

$$
e=-k_{1} y_{1}-k_{2} v_{2}
$$

Now

$$
\operatorname{Var}\{e]=\sigma_{e}^{2} \triangleq E\left[e^{2}-E[e]^{2}\right\}
$$

which in view of the unbiasedness of $e$ becomes:

$$
\sigma_{e}^{2}=E\left[e^{2}\right]
$$

Substitution of (10) into (12) yields:

$$
\sigma_{e}^{2}=E\left(k_{1}^{2} v_{1}^{2}+k_{2}^{2} v_{2}^{2}+2 k_{1} k_{2} v_{1} v_{2}\right\}
$$

Using (2.b,d,e) and (8), (13) becomes:

$$
\sigma_{e}^{2}=\left(1-k_{2}\right)^{2} \sigma_{1}^{2}+k_{2}^{2} \sigma_{2}^{2}
$$

We want the estimator to be of minimum variance. From (14) we see that we still haqve one more design parameter to choose; namely $k_{2}$. Consequently, we choose $k_{2}$ so as to minimize $\sigma_{e}^{2}$. Searching for the minimum we differentiate (14) with respect to $k_{2}$ and equate the result to zero. This yields:

$$
\frac{d}{d k_{2}}\left(\sigma_{e}^{2}\right)=-2\left(1-k_{2}\right) \sigma_{1}^{2}+2 k_{2} \sigma_{2}^{2}=0
$$

Consequently:

$$
k_{2}=\frac{\sigma_{1}^{2}}{\sigma_{1}^{2}+\sigma_{2}^{2}}
$$

It can be easily verified that the stationary point which $\sigma_{e}^{2}$ has at this $k_{2}$, is a minimum point. Using (16.a) in (8), we obtain: 


$$
k_{1}=\frac{\sigma_{2}^{2}}{\sigma_{1}^{2}+\sigma_{2}^{2}}
$$

Substitution of (16) into (4) yields:

$$
\hat{x}=\frac{\sigma_{2}^{2}}{\sigma_{1}^{2}+\sigma_{2}^{2}} y_{1}+\frac{\sigma_{1}^{2}}{\sigma_{1}^{2}+\sigma_{2}^{2}} y_{2}
$$

\section{Q.E.D.}

\section{References}

1. Black, H.D., "A Passive System for Determining the Attitude of a Satellite," AIAA Journal, Vol. 2, No. 7, July 1964, pp. 1350, 1351.

2. Shuster, M.D., and Oh, S.D., "Three-Axis Attitude Determination from Vector Observations," Journal of Guidance and Control, Vol. 4, No. 1, Jan.-Feb, 1981, pp. 70-77.

3. Bar-Itzhack, I. Y., and Meyer, J., "On the Convergence of Iterative Orthogonalization Processes," IEEE Transactions on Aerospace Electronic systems, Vol. AES-12, No. 2, March 1976, pp. 146-151. 
